\title{
Carnivores are right we must eat meat
}

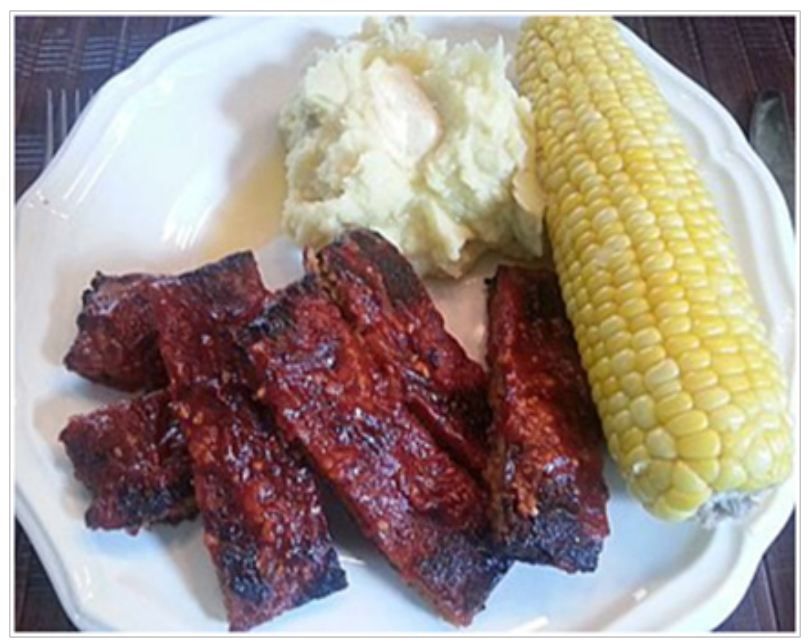

\section{Opinion}

It's true. There is no way the planetary population could just give up eating animals overnight. They would all starve! Even those addicted to meat that are able to eat alternative Foods would pretty much starve because they just don't understand or know how to prepare alternative Foods. Alternative Foods are basic Plant Based vegetables, fruits, nuts and seeds. Pretty simply huh? But other than eating an apple or banana or a big salad, what else is available? This is where the disconnect occurs in almost every Country on the Planet and amongst almost every population center on Earth!

Plant Based folks and Vegans all proclaim that eating animals is bad or wrong and can give all sorts of vivid, and often disgusting, examples of their point of view. Animal rights vegans proclaim that eating animals and raising them for food is cruel; well maybe it is but that is not the point. Plant Based folks proclaim (like I often do) that consuming animal based foods is detrimental to your health \& wellness and that living a Plant Based Lifestyle can often prevent and Reverse many lifestyle diseases (heart disease, diabetes, cancer and more).

They miss the point. The Planet is not capable, in its current state, to Feed all the people of the Earth with just vegetables, fruits, nuts and seeds; there just isn't enough currently plant foods produced or available to provide for the hungry masses if meat products were not available. I think this is tragic but face the Fact that it is true! Producing animals for food and feeding millions has been a Planetary focus for too long and meat consumption has continued its dramatic rise; especially over the past few decades!

Here are some quick statistics (and yes they are supportable):

i. Animal Agriculture is responsible for $18 \%$ of greenhouse gas emissions; more than the combined exhaust from ALL transportation.

\author{
Volume 5 Issue I - 2016
}

\section{Skip Stein}

Plant Based Lifestyle Consultant, Whole Foods 4 Healthy Living, USA

Correspondence: Skip Stein, Plant Based Lifestyle Consultant, Chief Operating Officer, Whole Foods 4 Healthy Living, Orlando, Florida, USA, Tel 40768368I6,

Email skipstein@wholefoods4healthyliving.com

Received: October 01, 2016 | Published: October 21, 2016

ii. Livestock and their by products account for at least 32,000 million tons of $\mathrm{CO}_{2}$ per year; that is $51 \%$ of ALL worldwide greenhouse gas emissions.

iii. Cows produce 150 billion gallons of methane per day and $65 \%$ of all human-related emissions of nitrous oxide.

iv. Animal Agriculture consumes from 34 to 76 trillion gallons of water annually. Franking only uses about 70billion gallons annually.

v. Feed crops for livestock consume $56 \%$ of all water used in the USA.

vi. More than 2,500 gallons of water are needed to produce 1pound of beef and it takes 14 to 16 months of care and feeding before a cow can be slaughtered for meat.

If anyone with basic accounting skills could determine, raising animals for food is not cost effective. Animal farming produces detrimental side effects that harm the planet and those who consume the animal products. This way of feeding the planet with meat is unsustainable and is in the process of collapsing as the environment is destroyed and people continue to ever sicken and die. Then there are the rising prices of animal foods. Vegetables are still cheap compared to meat products as are fruits, nuts and seeds but we still don't produce enough to replace all the animal protein; even though, pound for pound most vegetables have More protein and vital nutrients.

So, forgetting the addiction that is caused by consuming meat, fish, dairy and eggs (and Yes it is addictive), the only way we can survive is to move towards a Plant Based Sustainable Lifestyle. This means a reduction in animal and processed foods, reducing the plants grown for animal feed and start growing vegetables and human edible plants.

We as a species must move away from processed animal products as food and towards healthier and Delicious Whole Foods grown in the 
soil (or hydroponically), not animals raised in factory farms. Whole Food Real Food that is natural, whole and Delicious and Nutritious is the only way we can heal ourselves and survive!

\section{But that will never work!}

Why, simply because most people just don't understand or want to learn HOW to prepare (yes we mean going into the most unused part of many homes) Food in the Kitchen! Yes, the verboten word and area most feared in many/most American homes. There I said it: Kitchen!

The crazy but fascinating thing is that you can save a Lot of Money that way, $30 \%$ or more by preparing your own meals in the Kitchen. No it doesn't take a lot of skill or time; otherwise humans would have gone extinct eons ago. Cooking Whole Food meals is as natural as breathing and just as necessary! The Fear is that too many people truly believe that eating and living a Plant Based Lifestyle is somehow less delicious or nutritious. People continue to believe (fed by endless commercials by the meat/dairy industry) that animal protein is somehow necessary; when the simple Fact is that animal protein is one of the leading causes of Death!
Just try a simple, low cost, experiment. Try to prepare a meal in your Kitchen. Need a recipe? No need to spend a penny, just visit: https://www.pinterest.com/Gramstein/Chef Nancy Stein has over 20,000 FREE and delicious recipes posted and is available to help you over your initial fear!

Give it a try for 30 days and see if you don't feel better, have more energy, feel less depressed, feel more vital and find that Food can be Delicious. You will not only achieve improved health but you will learn the marvellous flavours, textures and smells that delicious Plant Based Cuisine can offer!

\section{Acknowledgements}

None.

\section{Conflict of interest}

Author declares that there is no conflict of interest. 